\title{
In vitro and in vivo antitumor effects of 50 to 100-KDa components from B16 melanoma culture supernatant
}

\author{
YING-SONG QIN $^{1}$, XU ZHANG ${ }^{2}$ and XIANG-YU ZHANG ${ }^{3}$ \\ ${ }^{1}$ Department of Pathophysiology, Xuzhou Medical College; ${ }^{2}$ Department of Otolaryngological, \\ Affiliated Hospital of Xuzhou Medical College, Xuzhou, Jiangsu; ${ }^{3}$ State Key Laboratory of Oncogenes and Related Genes, \\ Shanghai Cancer Institute, Renji Hospital, School of Medicine, Shanghai Jiao Tong University, Shanghai, P.R. China
}

Received March 7, 2014; Accepted December 16, 2014

DOI: $10.3892 / 01.2015 .3239$

\begin{abstract}
The development of immunological therapies for melanoma has been of considerable concern in recent years. Whole tumor cell lysates have been used to develop antitumor vaccines, but the effective components of the lysates have not been identified. In the present study, protein elements were purified from the B16 supernatant to analyze the in vitro chemotaxis towards mouse spleen lymphocytes using a Boyden chamber. Prior to establishing a B16 melanoma model, C57BL/6 mice were vaccinated with these proteins, and melanoma growth, tumor appearance time and behavioral changes were observed. Next, the cytotoxicity and subsets of the tumor infiltrating lymphocytes, and the histological characteristics of the melanoma were analyzed. The isolated purified fragments of B16 melanoma culture supernatant had strong antitumor effects. The possible antitumor mechanism was delineated, and was identified to possibly be through the activation of cluster of differentiation 8-positive $\mathrm{T}$ cells and the promotion of B16 cell differentiation. These methods will provide a novel insight into understanding antitumor immunological mechanisms and provide a potential avenue for immunotherapy.
\end{abstract}

\section{Introduction}

The B16 melanoma cell exhibits low immunogenicity and lacks a major histocompatibility complex-I molecule. The transplanted B16 melanoma mouse model is widely used to study the immunology and immunological escape of tumor cells (1). In the advanced stage, transplanted B16 melanomas almost always develop lung metastases. Antitumor immunology has been widely studied, including the use of tumor

Correspondence to: Dr Xiang-Yu Zhang, State Key Laboratory of Oncogenes and Related Genes, Shanghai Cancer Institute, Renji Hospital, School of Medicine, Shanghai Jiao Tong University, 84 Huaihai West Road, Shanghai 200032, P.R. China

E-mail: zhangxiangyu666@126.com

Key words: chemotaxis, immune killing, tumor infiltrating lymphocyte, vaccination, culture supernatant vaccines, adoptive lymphocyte treatment and tumor-frozen treatment (2). These tactics share the same mechanism of activating the immunological cells to kill the tumor cells. The immunological cells, including macrophages, $\gamma \delta \mathrm{T}$ lymphocytes, cytotoxic lymphocytes and the adoptively transferred tumor-specific lymphocytes may elicit graft-versus-host disease, although the possibility is extremely low (3). The activated cells achieve their functions by direct interaction with the tumor cells, such as through the use of the Fas/FasL killing mechanism, or by indirect interaction with the tumor cells (4). The immunological cells usually secrete a number of cytokines, including tumor necrosis factor- $\alpha$, interferon and interleukin (IL)-12, which often induce tumor cell apoptosis or necrosis (5).

The use of tumor vaccines as tumor therapy has been explored for numerous decades, and a number of positive results have been achieved, particularly in combination with other therapeutics. A number of vaccines can markedly activate the host immune system to kill the tumor cells, such as the BORIS-based vaccine, which has been shown to increase effector cluster of differentiation $(\mathrm{CD}) 4^{+}$and $\mathrm{CD}^{+} \mathrm{T}$ cell infiltration towards a 4T1 mammary implanted tumor (6). Another common method is the use of dendritic cells (DCs), the most potent antigen-presenting cells, which can stimulate the T cells. As DCs can be loaded with a number of varying types of antigen, DCs can effectively stimulate cytotoxic T lymphocytes (CTL) (7). Recently, vaccination techniques have been combined with nanotechnology to synthesize antitumor vaccines that target certain tumor cellular antigens. The approach stimulates the body to generate long-lasting antibodies, and these antibodies can selectively target the antigens in the tumor cells, resulting in eventual tumor cell death (8). Whole tumor cell vaccines have shown great potential with regard to their antitumor effects; irradiated tumor cells pulsed with an adjuvant can stimulate the $\mathrm{CD}^{+} \mathrm{T}$ cell-mediated adaptive immune response $(9,10)$, while another strategy is to fuse the tumor cell lysate with dendritic cells, which have robust efficacy in expanding antigen-specific $\mathrm{CD}^{+} \mathrm{T}$ cells (11). Formalin-fixed B16 cells together with IL-12 show a strong antitumor response, which is mediated by $\mathrm{CD}^{+}$and $\mathrm{CD}^{+} \mathrm{T}$ cells (12). The use of whole tumor cells as antitumor vaccines is effective in activating tumor infiltrating lymphocytes (TILs), and as the tumor 
cells contain various known and unknown antigens, besides proteins, they can cause a number of side-effects, including inflammatory reactions (13). When whole tumor cell lysates are fused with DCs, the whole vaccines can exert much strong antitumor effects (14). However, the effective components of whole tumor cell lysates have not been fully investigated and remain unclear to a certain extent. An ideal vaccine remains to be discovered or engineered.

TILs have long been considered as the main effector of antitumor immune responses (15). The presence of TILs in human cancers shows that the immune system recognizes the tumor to a certain degree. Studies have shown the benefit of TILs in human cancers, particularly with regard to the number of $\mathrm{CD}^{+} \mathrm{T}$ cells $(16,17)$. TILs contain numerous lymphocytes, including $\mathrm{CD}^{+} \mathrm{T}$ cells, $\mathrm{CD} 8^{+} \mathrm{T}$ cells and $\mathrm{CD} 20^{+} \mathrm{B}$ cells. However, a number of studies have indicated that the activated $\mathrm{CD}^{+} \mathrm{T}$ cells are the major functioning cells in an antitumor immunological reaction. $\mathrm{CD} 8^{+} \mathrm{T}$ cells can induce tumor cell apoptosis or tumor rejection through direct or indirect contact with targeted tumor cells. Previous studies have suggested that high levels of TILs are correlated with a better prognosis (17). CTLs are crucial in antitumor immune responses $(18,19)$; $\mathrm{CD}^{+}{ }^{+} \mathrm{T}$ cells aid in the activation of $\mathrm{CD}^{+} \mathrm{T}$ cells and then induce $\mathrm{CD}^{+} \mathrm{T}$ cells to kill the tumor cells. Although $\mathrm{CD}^{+} \mathrm{T}$ cells are not the main effectors, they are often indispensable in this process. Studies concerning $\mathrm{CD}^{+} / \mathrm{CD} 25^{+} / \mathrm{Foxp}^{+}{ }^{+}$Treg cells have also become a focus of attention. These cells usually have negative regulatory effects in antitumor responses. Treg can suppress the antitumor immune responses and maintain the state of immunological unresponsiveness during the process (20).

In the present study, the B16 melanoma culture supernatant was isolated and the isolated purified fragments were used to determine the in vitro and in vivo antitumor effects, with the aim of identifying potential novel treatment avenues for melanoma.

\section{Materials and methods}

Materials. C57BL/6 mice, 8-10 weeks old and weighing 18-20 g, were obtained from Xuzhou Medical College Experiment Animal Center (Xuzhou, Jiangsu, China). All surgical procedures and normal experimental processes were performed in accordance with the guidelines of the Xuzhou Medical College Animal Care and Use Committee. This study was approved by the ethics committee of Xuzhou Medical College. B16 melanoma cells were purchased from Shanghai Institutes for Life Science, the Chinese Academy of Sciences (Shanghai, China). The B16 cells were syngeneic with the C57BL/6 mice used for the vaccination. The B16 cells were cultured with RPMI 1640 medium supplemented with $10 \%$ heat inactivated fetal bovine serum (FBS) (Zhejiang Tianhang Biological Technology Co., Ltd., Hangzhou, China), $100 \mathrm{U} / \mathrm{ml}$ penicillin and $100 \mu \mathrm{g} / \mathrm{ml}$ streptomycin. Fluorescein isothiocyanate (FITC)-conjugated anti-mouse CD4 antibody, FITC-conjugated anti-mouse CD8a antibody and phycoerythrin (PE)-conjugated anti-mouse CD20 antibody were purchased from BioLegend (San Diego, CA, USA), and PE-conjugated anti-mouse CD20 was purchased from eBioscience (San Diego, CA, USA). Cell counting kit-8, used to determine the cytotoxicity of the TILs, was purchased from Beyotime Institute of Biotechnology (Haimen, Jiangsu, China), and EZ-Sep ${ }^{\mathrm{TM}}$ Mouse 1X Lymphocyte Separation Medium was purchased from Dakewe Biotech Company (Shenzhen, Guangdong, China).

Selective isolation of B16 melanoma supernatant elements and preparation of mouse spleen lymphocytes. The B16 cells were cultured as previously reported (21). Briefly, B16 cells were cultured in RPMI 1640 medium (Gibco Life Technologies, Carlsbad, CA, USA). After $48 \mathrm{~h}$, the medium was collected and centrifuged at $10,000 \mathrm{x}$ g at $4^{\circ} \mathrm{C}$ for $10 \mathrm{~min}$. The supernatant was then collected for analysis of its elements in an ultrafiltration centrifuge tube. The isolated elements consisted of fragments with sizes of 3-5, 5-10, 10-30, 30-50, 50-100, 100-300 and $>300 \mathrm{KDa}$. A C57BL/6 mice were euthanized and then the spleen was dissected, minced into small pieces, passed sequentially through cell strainers $(40 \mu \mathrm{m})$ and washed in RPMI 1640 without FBS.

The 50-100 and 100-300-KDa molecular weight fractions, original supernatant and RPMI 1640 medium were used to carry out the chemotaxis experiment in a Boyden chamber. The lymphocytes were placed in the lower compartment and the aforementioned elements were placed in the upper chamber. Quantification of the number of lymphocytes in the lower compartment was performed at the $0.5,2,4,8,12$ and 24-h time-points.

Immunizations and analyses of tumor growth or lung metastases. Five groups of C57BL/6 mice ( $\mathrm{n}=8$ per group) were subcutaneously (s.c.) injected into the right flanks twice with the 50-100-KDa molecular weight fraction, the 100-300-KDa molecular weight fraction, repeatedly freeze-thawed B16 melanoma cells, original supernatant or RPMI 1640 medium. After two weeks, $200 \mu \mathrm{l}$ B16 cells at a concentration of $1 \times 10^{6} \%$ $\mathrm{ml}$ were injected s.c. at day 14 into the right armpit of the mice. Tumor growth was monitored daily once the tumor became palpable at day 20, six days after B16 cell implantation. The tumor volume was determined by two-dimensional measurements and calculation using the formula $\left(\mathrm{a} \mathrm{x} \mathrm{b}^{2}\right) / 2$, where a represents the largest diameter and $b$ the smallest diameter of the tumor. On day 21 post-tumor implantation, all the mice were sacrificed and the number of lung metastases in the lungs were counted, and the tumor and lung pathology characteristics were analyzed using hematoxylin and eosin (HE) staining.

Cytotoxicity of TILs and splenic lymphocytes (SPLs). On day 21 , the tumor and spleen were surgically removed from each tumor-bearing mouse, and TILs and SPLs were isolated as aforementioned. The TILs were mixed with the B16 cells at effector:target (E:T) ratios of 12.5:1, 25:1 and 50:1. The cytotoxicity of the TILs was determined in a 96-well plate using a CCK-8 kit, and each experiment was repeated three times. The cytotoxicity of the SPLs was determined using the same method.

Analysis of TIL and SPL populations. Once the tumor and spleen had been surgically removed from each tumor-bearing mouse, the tissues were each minced and digested in $1 \mathrm{mg} / \mathrm{ml}$ collagenase type IV, then subjected to filtration and washing. The cells were then stained with anti-CD4-FITC, 
Table I. B16 cell culture supernatant isolated purified fragment chemotaxis (mean \pm standard error of the mean; $\mathrm{n}=4$ ).

Time-points

\begin{tabular}{lcccccc}
\cline { 2 - 6 } Group & $0.5 \mathrm{~h}$ & $2 \mathrm{~h}$ & $4 \mathrm{~h}$ & $8 \mathrm{~h}$ & $12 \mathrm{~h}$ & $24 \mathrm{~h}$ \\
\hline $50-100 \mathrm{KDa}$ & $2.13 \pm 0.31$ & $5.50 \pm 0.20$ & $64.00 \pm 3.61$ & $103.33 \pm 5.86^{\mathrm{a}, \mathrm{b}}$ & $91.33 \pm 3.21^{\mathrm{a}, \mathrm{b}}$ & $95.00 \pm 2.00^{\mathrm{a}, \mathrm{b}}$ \\
$100-300 \mathrm{KDa}$ & $2.00 \pm 0.25$ & $5.07 \pm 0.40$ & $49.67 \pm 3.51$ & $78.33 \pm 5.69^{\mathrm{a}}$ & $77.33 \pm 2.52^{\mathrm{a}}$ & $79.00 \pm 2.65^{\mathrm{a}}$ \\
Original supernatant & $3.40 \pm 1.06$ & $9.67 \pm 2.08$ & $18.00 \pm 2.00$ & $47.67 \pm 2.08^{\mathrm{a}}$ & $47.33 \pm 1.53^{\mathrm{a}}$ & $48.33 \pm 1.53^{\mathrm{a}}$ \\
RPMI 1640 & $2.10 \pm 0.36$ & $3.50 \pm 0.50$ & $10.00 \pm 0.00$ & $17.00 \pm 2.00$ & $17.00 \pm 1.00$ & $18.00 \pm 1.00$
\end{tabular}

One-way repeated measures analysis of variance. ${ }^{\mathrm{a}} \mathrm{P}<0.05$ vs. $0.5 \mathrm{~h}$ and $2 \mathrm{~h}$ within each group; ${ }^{\mathrm{b}} \mathrm{P}<0.05$ vs. RPMI 1640 group.

anti-CD28-PE, anti-CD8-FITC, anti-CD28-PE and anti-CD20-PE antibodies. The obtained TILs and SPLs were each adjusted to $1 \times 10^{6} / \mathrm{ml}$. Following incubation with fluorescence antibodies for $30 \mathrm{~min}$ in the dark on ice, the cell suspensions were analyzed on a FACScan flow cytometer (Becton Dickinson, San Jose, CA, USA).

Statistical analysis. One-way repeated measures analysis of variance was used for the statistical analysis, and $\mathrm{P}<0.05$ was considered to indicate a statistically significant difference. The data were expressed as the means \pm standard error of the mean and were representative of three different experiments.

\section{Results}

Characterization of B16 cell culture isolated purified fragments. Subsequent to separation and purification, SDS-PAGE gel electrophoresis showed that the B16 cell culture supernatant was comprised of proteins with molecular weights of $\sim 70$ or $130-250 \mathrm{KDa}$, although much more of the $70-\mathrm{KDa}$ protein was present in comparison. No obvious bands were apparent in other lanes (Fig. 1).

Evaluation of chemotaxis of isolated purified fragments. With regard to the 50-100 and 100-300-KDa molecular weight fractions, the original supernatant and the RPMI 1640 medium, the chemotaxis of the four groups was enhanced with increasing chemotaxis time intervals. The quantity of lymphocytes attracted by the chemotaxis reached a summit at the 8-h time-point. At the 8-h time-point, the level of chemotaxis lymphocytes in the $50-100 \mathrm{KDa}$ group was the highest at $103.33 \pm 5.86 \times 10^{4} / \mathrm{ml}$, while the second highest level of $78.33 \pm 5.69 \times 10^{4} / \mathrm{ml}$ was found in the $100-300 \mathrm{KDa}$ group. These levels were significant compared with the other three groups, respectively, $(\mathrm{P}<0.05)$ (Table I).

Evaluation of therapeutic potency of isolated purified fragments. The separated and purified protein, repeatedly freeze-thawed B16 cells, original supernatant and RPMI 1640 medium were used to vaccinate five groups of C57BL/6 mice. All mice manifested no changes in living conditions. The tumors of all five groups of mice became palpable on day 6 post-transplantation; the tumor growth of the 50-100 KDa group was the slowest, while the repeatedly freeze-thawed B16 cell group was the second slowest among

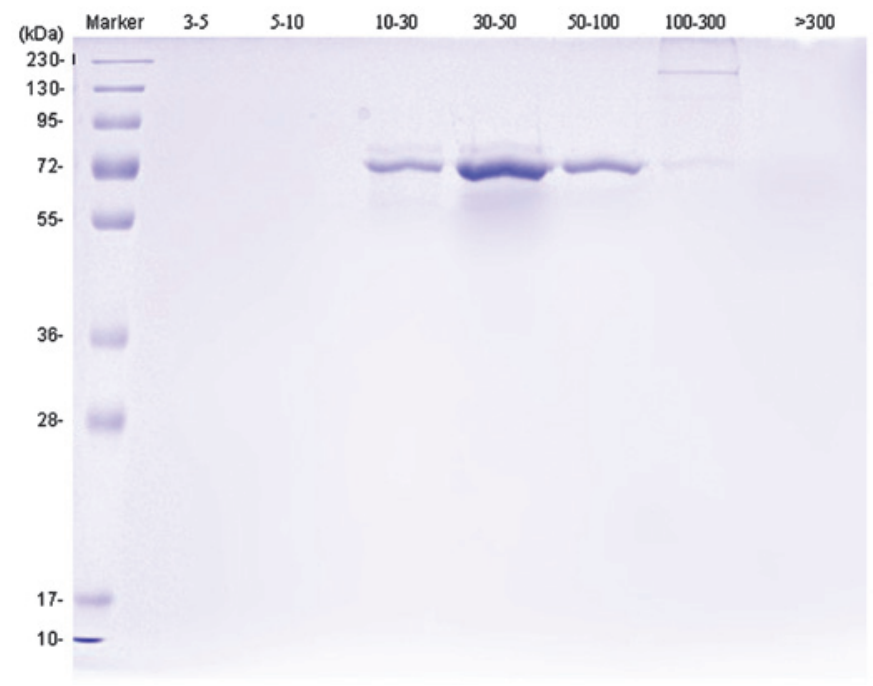

Figure 1. Identification of B16 cell culture supernatant isolated purified fragments by SDS-PAGE analysis. The protein is mainly distributed in the 10-30, $30-50$ and 50-100 lanes; the molecular weight of these proteins is $\sim 70 \mathrm{KDa}$.

the five groups. The tumors of the RPMI 1640 group grew the fastest compared with the 50-100 KDa group on the same day, and the difference was statistically different $(\mathrm{P}<0.05)$ (Fig. 2). On day 14, when the tumors were surgically removed from the tumor-bearing mice, the volume and weight of the tumors from the 50-100 KDa group was $520.15 \pm 36.69 \mathrm{~mm}^{3}$ and $1323.75 \pm 27.54 \mathrm{mg}$, respectively, which were the smallest measurements when compared with the other groups. The next smallest measurements were those of the repeatedly freeze-thawed B16 cell group, while the volume and weight of the tumors in the RPMI 1640 group was $2363.50 \pm 43.05 \mathrm{~mm}^{3}$ and $2593.75 \pm 95.65 \mathrm{mg}$, which were the largest measurements among all the groups $(\mathrm{P}<0.05)$. No accidental mortality occurred during this period (Fig. 3).

Cytotoxicity of TILs and SPLs. CCK-8 analysis determined that the cytotoxicity of the TILs was much higher than that in the corresponding SPLs within the same group, and the cytotoxicity increased as the E:T ratio increased. Among the five different groups, the cytotoxicity of the TILs and SPLs of the 50-100 KDa group was the highest at $0.82 \pm 0.026$ and $0.67 \pm 0.029$, respectively, which was statistically different compared with the four other groups $(\mathrm{P}<0.05)$ (Fig. 4). 

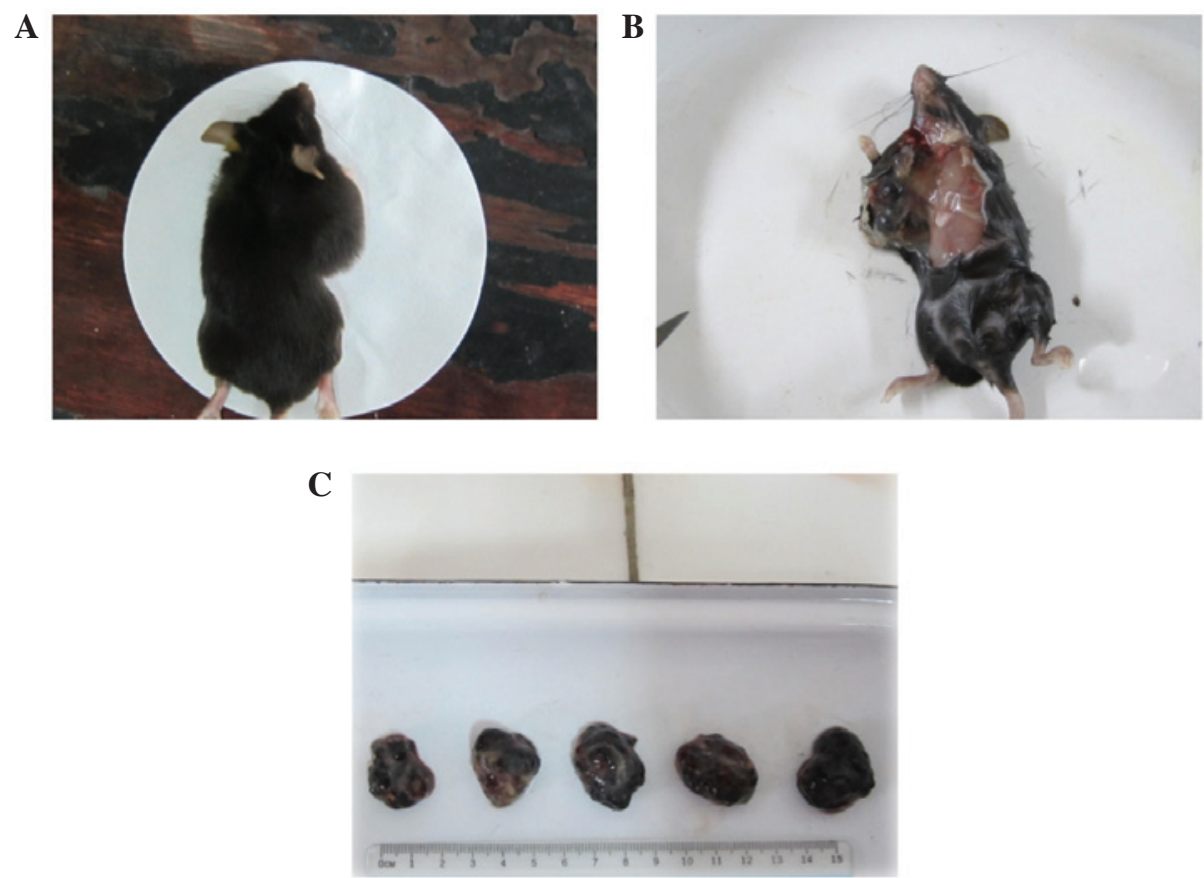

Figure 2. Establishment of a B16 melanoma mouse model and transplanted tumor growth. (A and B) The C57BL/6 mice were subcutaneously transplanted with B16 cells $(n=8)$. (C) The tumors were dissected and weighed at experiment termination; from the left to the right of the images are the 50-100 KDa group, the 100-300 KDa group, the repeatedly freeze-thawed B16 cell group, the original supernatant group and the RPMI 1640 medium group. The tumor of the 50-100 KDa group is the smallest among the five groups.
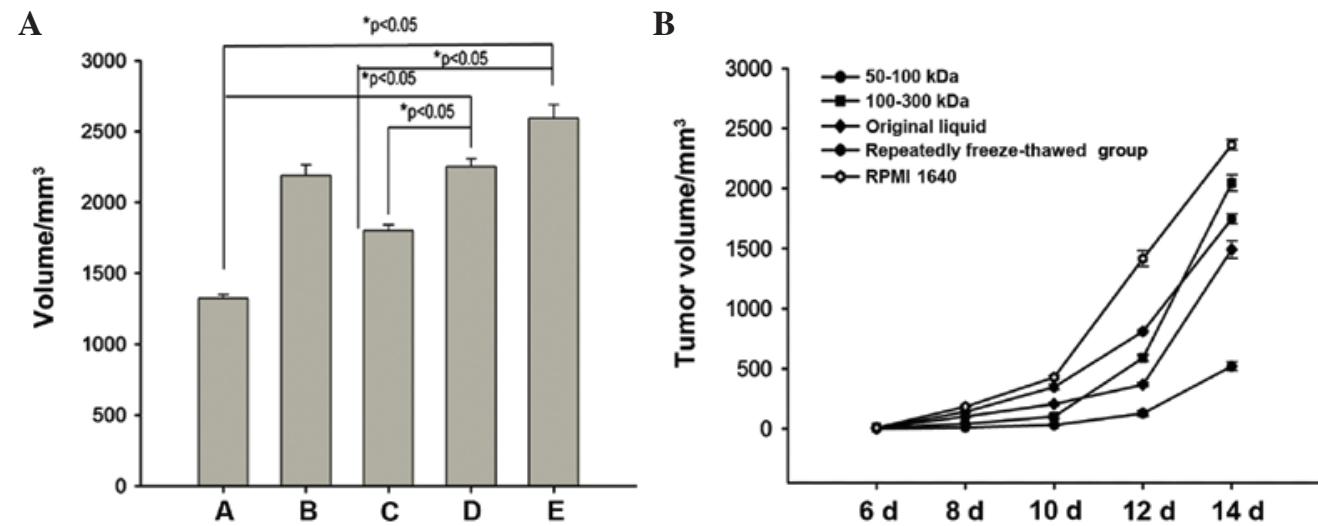

Figure 3. Growth curves of (A) B16 melanomas vaccinated with 50-100 KDa molecular weight fractions, 100-300 KDa molecular weight fractions, repeatedly freeze-thawed B16 melanoma cells, original supernatant and RPMI 1640 medium. The 50-100 KDa group exhibited the lowest tumor volume, while the RPMI 1640 medium group exhibited the highest. A, 50-100 KDa group; B, 100-300 KDa group; C, repeatedly freeze-thawed B16 cell group; D, original supernatant group; E, RPMI 1640 medium group. (B) Histogram showing the average weight of the dissected tumors from the five different groups (n=8). Data in (A) and (B) are representative of results from two separate experiments with eight mice/group in each experiment (bars represent the standard deviation: ${ }^{*} \mathrm{P}<0.05$ relative to the same group on day $6,{ }^{*} \mathrm{P}<0.05$ relative to RPMI 1640 medium group and ${ }^{*} \mathrm{P}<0.05$ relative to original supernatant group).

Lymphocyte subsets in transplanted B16 melanomas and mouse spleens. The tumors and spleens were surgically removed from the mice, then subjected to analysis of their activated $\mathrm{CD} 4^{+} \mathrm{T}$ cells, activated $\mathrm{CD} 8^{+} \mathrm{T}$ cells and $\mathrm{CD} 20^{+} \mathrm{B}$ cells using flow cytometry. In each respective group, the percentage of activated $\mathrm{CD} 8^{+} \mathrm{T}$ cells from the TILs was much higher than the percentage from the SPLs, with the exception of the RPMI 1640 group. Among the five different groups, the percentage of activated $\mathrm{CD} 8^{+} \mathrm{T}$ cells from the corresponding TILs was markedly higher in the 50-100 KDa group (39.61\%) compared with the other four groups. The second highest percentage was found in the
100-300 KDa group, while the RPMI 1640 group exhibited the lowest percentage of activated $\mathrm{CD} 8^{+} \mathrm{T}$ cells. The percentage of activated $\mathrm{CD} 4^{+} \mathrm{T}$ cells from the corresponding TILs was markedly higher in the 50-100 KDa group (47.76\%) compared with the other four groups, while the RPMI 1640 group exhibited the lowest percentage. With regard to the SPL subsets, the percentage of activated $\mathrm{CD} 8^{+} \mathrm{T}$ cells was the highest in the freeze-thawed group (18.7\%), while the 50-100 KDa group exhibited the lowest percentage. The percentage of activated $\mathrm{CD} 4^{+} \mathrm{T}$ cells was the highest in the original supernatant group (19.8\%) and the lowest in the 50-100 KDa group (Table II). 

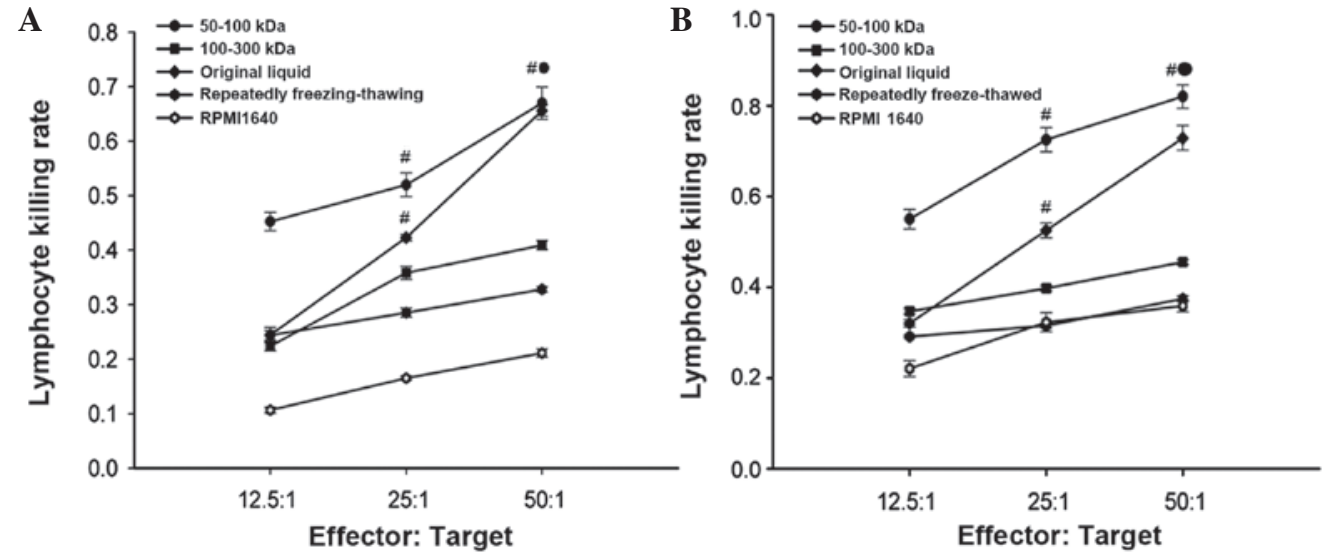

Figure 4. Cytotoxicity of tumor-infiltrated lymphocytes (TILs) and splenic lymphocytes (SPLs) in the 50-100 and 100-300-KDa molecular weight fractions, the repeatedly freeze-thawed B16 melanoma cells, the original supernatant and the RPMI 1640 medium immunized mice. (A) Cytotoxicity of SPLs from the same group increases as the E:T ratio augments; the cytotoxicity of the 100-300 KDa group is the most effective at the same E:T ratio when compared with the other four groups; (B) Cytotoxicity of TILs from the same group increases as the E:T ratio augments; cytotoxicity of the 100-300 KDa group is the most effective at the same E:T ratio compared with the other four groups. (bars represent the standard deviation: ${ }^{\#} \mathrm{P}<0.05$ relative to the same group at the E:T ratio of $12.5,{ }^{\bullet} \mathrm{P}<0.05$ relative to the RPMI 1640 medium group at the same E:T ratio).

A

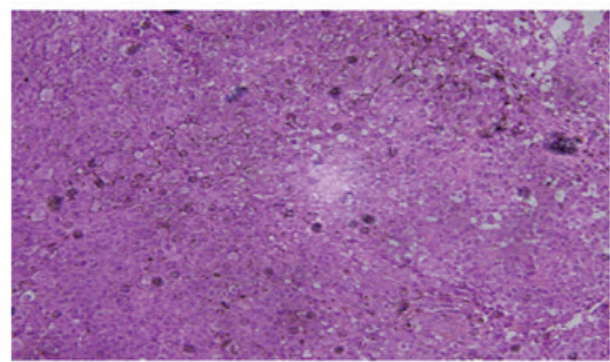

C
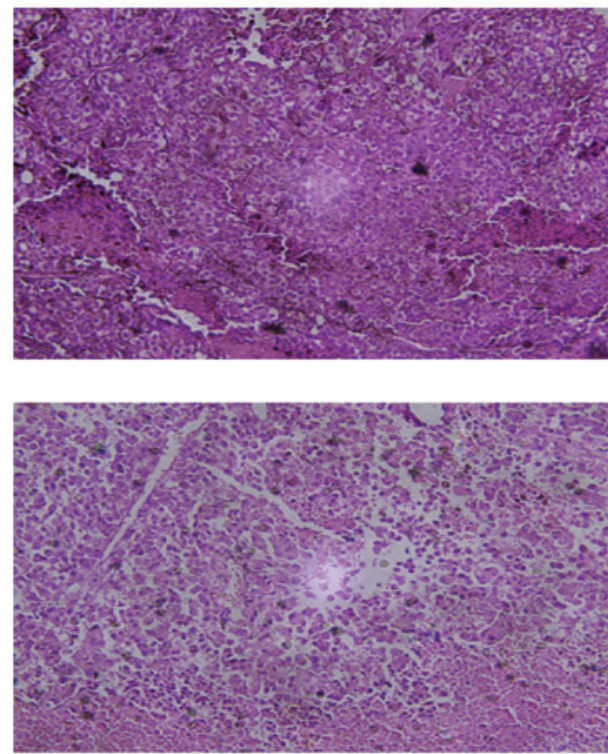
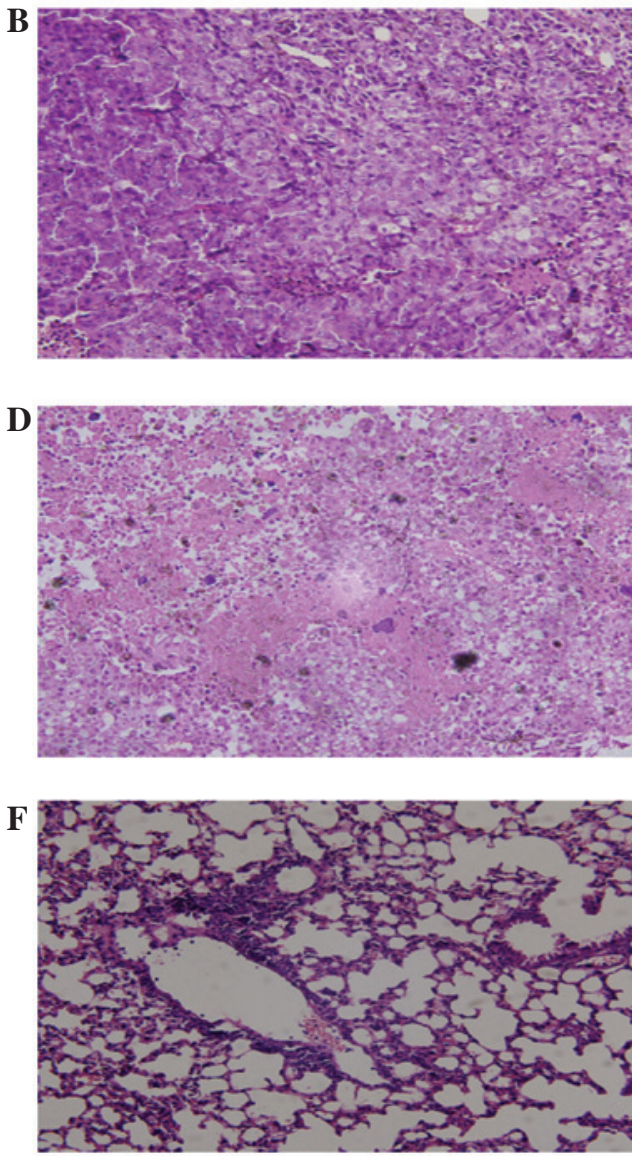

Figure 5. Hematoxylin and eosin staining of the transplanted tumor and mouse lungs. The tumor cells of mice vaccinated with (A) 50-100-KDa molecular weight fractions, (B) 100-300 KDa molecular weight fractions, (C) repeatedly freeze-thawed B16 melanoma cells, (D) original supernatant and (E) RPMI 1640 medium, showin a certain degree of aberrant differentiation. However, the atypia of (A) is much less evident than that of the mice vaccinated with RPMI 1640 medium. (F) Mouse without lung metastasis. Original magnification, x200.

Histological characteristics of the transplanted tumor and lung. Histological characteristics were assayed using HE staining, which revealed no lung metastasis in any group. However, local axillary lymph node metastasis was observed in each group. The number of metastatic lymph nodes was the highest in the RPMI 1640 group and the lowest in the 50-100 KDa group. Hemorrhage and necrosis of the transplanted tumor only occurred in the RPMI 1640 group. Using microscopy, a large degree of cytologic atypia was apparent in several of the groups, particularly the RPMI 1640 group. 
Table II. Lymphocytes subsets percents in TIL and SP.

\begin{tabular}{|c|c|c|c|c|c|}
\hline \multirow[b]{2}{*}{ Group } & \multicolumn{3}{|c|}{ TIL, \% } & \multicolumn{2}{|c|}{ SPL, $\%$} \\
\hline & $\mathrm{CD} 28^{+} / \mathrm{CD} 4^{+} \mathrm{T}$ cells & $\mathrm{CD} 28^{+} / \mathrm{CD} 8^{+} \mathrm{T}$ cells & $\mathrm{CD} 20^{+} \mathrm{B}$ cells & $\mathrm{CD} 28^{+} / \mathrm{CD} 4^{+} \mathrm{T}$ cells & $\mathrm{CD} 28^{+} / \mathrm{CD} 8^{+} \mathrm{T}$ cells \\
\hline A & 47.76 & 39.61 & 35.60 & 10.19 & 6.64 \\
\hline $\mathrm{B}$ & 25.10 & 32.30 & 34.80 & 15.7 & 17.9 \\
\hline $\mathrm{C}$ & 13.80 & 26.60 & 38.40 & 17.7 & 18.7 \\
\hline D & 15.00 & 17.90 & 34.3 & 19.8 & 15.6 \\
\hline $\mathrm{E}$ & 8.94 & 10.20 & 36.1 & 16.0 & 12.2 \\
\hline
\end{tabular}

A, B , C , D, E represents 50-100 KDa group, 100-300 KDa group, repeatedly freezing-thawing group, original supernatant group and RPMI 1640 medium group, respectively. Results are from one of the three independent experiments. TIL, tumor infiltrating lymphocytes; SPL, splenic lymphocytes.

The tumor cells were also observed to have matured and differentiated dysfunctionally; this was particularly evident in the RPMI 1640 group, while the 50-100 KDa group was much less affected (Fig. 5).

\section{Discussion}

In order to define the functions of TILs, B16 cell supernatant isolated purified fragments were used to analyze the hypothesized antitumor immunological reactions. It was found that the protein molecular weight of the B16 cell culture supernatant was $\sim 70 \mathrm{KDa}$, and that the 50-100 KDa group exhibited strong in vitro chemotaxis. When C57BL/6 mice were vaccinated with this fragment, it was able to markedly inhibit tumor growth. In addition, the cytotoxicity of the TILs and SPLs from this group was higher in comparison to the other examined groups. As $\mathrm{T}$ cell immunity was considered to be much more important than B cell immunity, the present study mainly analyzed the $\mathrm{T}$ cell subsets. The $\mathrm{CD} 28^{+} / \mathrm{CD} 4^{+} \mathrm{T}$ cell percentages of TILs from the 50-100 KDa group were the highest. The antitumor effects of 50-100 KDa molecular weight fractions may be attributable to the activation of $\mathrm{CD} 8^{+} \mathrm{T}$ cells, and the enhanced cytotoxicity of $\mathrm{CD} 8^{+} \mathrm{T}$ cells may also relate to the promotion of differentiation effects.

The results presented in the current study demonstrate an involvement of isolated purified fragments in initiating the in vitro chemotaxis and in vivo antitumor immunological reactions, leading to the marked growth inhibition of transplanted B16 melanoma. Taken together, these in vitro and in vivo experiments indicate that the antitumor effects of B16 cell supernatant isolated purified fragments are selective, and that the 50-100-KDa molecular weight fraction has the most potent effects among all the fractions, with the ability to activate $\mathrm{CD} 8^{+} \mathrm{T}$ cells and enhance the cytotoxicity of TILs for B16 cell killing. Tumor vaccines represent an effective antitumor immunotherapy, with ideal effects being the elimination of occult micro-metastases (6). However, a number of tumor vaccines are effective in animal models, but their effects in human tumors are not as expected $(22,23)$. Whole tumor cell-based vaccinations are multivalent and can elicit a broad range of responses to tumor-associated antigens, which are more potent than a single defined tumor antigen (24).
However, whole cell tumor vaccines consist of numerous cell elements, including lysosomal enzyme, proteolytic enzyme, cytochrome enzyme and heat shock protein. These elements, particularly the different types of enzymes, when administrated into the host can induce serious inflammation responses (25). However, the supernatant isolated purified fragments are selective proteins, and they can exert a strong antitumor immune response without being detrimental to the host (26). The present results also indicated that the cytotoxicity of the TILs and SPLs from the mice vaccinated with the 50-100-KDa molecular weight fraction was much stronger than that of repeatedly freeze-thawed B16 cells, which is in agreement with our hypothesis.

The culture supernatant isolated purified fragments are able to accumulate at a high concentration, which is required in the vaccination of mice, and most importantly, the fragments are not one protein, but several proteins with the approximate molecular weight. The present study is the first to report the antitumor effects of culture supernatant isolated purified fragments. The tumor antigens are also variable and tumor cell killing requires activation of different types of $\mathrm{T}$ cells (27). Taken together with the greater percentage of $\mathrm{CD} 28^{+} / \mathrm{CD}^{+} \mathrm{T}$ cells in the TILs of the $50-100 \mathrm{KDa}$ molecular weight fraction vaccinated mice, we speculate that the supernatant isolated purified fragments, particularly the 50-100 KDa molecular weight fraction, can mimic the tumor antigen to activate $\mathrm{CD} 8^{+} \mathrm{T}$ cells. Furthermore these $\mathrm{CD} 28^{+} / \mathrm{CD} 8^{+} \mathrm{T}$ cells have great in vitro cytotoxicity towards B16 cells, indicating that vaccinating mice with a 50-100 KDa molecular weight fraction can induce a $\mathrm{T}$ cell immune response and kill the tumor cells, eventually making the tumor mass disappear. Notably, the present study selected two B16 cell culture supernatant isolated purified fragments, as we have previously shown that the 50-100 and 100-300-KDa molecular weight fractions have potent chemotaxis (Qin et al unpublished data). The present results demonstrate that the antitumor immune response is associated with molecular weight culture supernatant isolated purified fragments.

Although the present study revealed the antitumor effects of B16 cell culture supernatant isolated purified fragments, the definitive protein involved and the types of protein are as 
yet unknown. Heat shock protein 70 (Hsp70) is a molecular chaperon, and the intratumoral application of Hsp70 on the surface of B16F10 melanoma tumors has been shown to reduce the tumor growth rate and prolong animal survival times (28); we propose that Hsp70 may be part of an isolated purified fragment, however, further biotechnological analysis is required to confirm this. Certain membrane proteins that have a molecular weight of $\sim 70 \mathrm{KDa}$ also require evaluation. A possible mechanism underlying the antitumor effects is the activation of $\mathrm{CD}^{+} \mathrm{T}$ cell subsets of TILs and the enhancement of the cytotoxicity of activated $\mathrm{CD} 8^{+} \mathrm{T}$ cells, all without changing the humoral immune response. The present results are consistent with other studies showing that the adaptive cellular immune response exerts a more significant role than the humoral immune response (28). In the present study, there were no lung metastases in any of the mice, but this is probably due to the limited growth time of the B16 melanoma. If the growth time had been extended then the metastases probably would have appeared. HE staining observations indicated that the tumor cells from the mice vaccinated with the 50-100-KDa molecular weight fraction exhibited more evident differentiation than the other four groups, suggesting that the 50-100-KDa molecular weight fraction can activate the unknown mechanism to promote B16 cell differentiation.

In conclusion, the present study firstly showed the chemotaxis and antitumor effects of B16 cell culture supernatant isolated purified fragments. The possible mechanism of these effects is also discussed. Mice vaccinated with the 50-100-KDa molecular weight fraction showed strong antitumor effects and marked inhibition of tumor growth compared with the repeatedly freeze-thawed B16 cells and 100-300-KDa molecular weight fraction. Frequencies of activated $\mathrm{CD}^{+}$conventional $\mathrm{T}$ cells with a Th1 profile were increased in the transplanted tumor, and the cytotoxicity of the activated $\mathrm{CD}^{+}$TILs was also increased. Taken together, these data indicate that the novel utilization of culture supernatant isolated purified fragments can effectively retard tumor growth. The present study provides an excellent platform on which to build effective antitumor therapeutics.

\section{Acknowledgements}

The authors would like to thank Professor Hong Liu of the Department of Pathology, Xuzhou Medical College, for providing technical assistance and valuable comments.

\section{References}

1. Gyorki DE, Callahan M, Wolchok JD and Ariyan CE: The delicate balance of melanoma immunotherapy. Clin Transl Immunology 2: e5, 2013.

2. Yee C, Thompson J, Byrd D, et al: Adoptive T cell therapy using antigen-specific CD8+ T cell clones for the treatment of patients with metastatic melanoma: In vivo persistence, migration, and antitumor effect of transferred T cells. Proc Natl Acad Sci USA 99: 16168-16173, 2002.

3. Cui NP, XIE SJ, Han JS, et al: Effective adoptive transfer of haploidentical tumor-specific T cells in B16-melanoma bearing mice. Chin Med J (Engl) 125: 794-800, 2012.

4. Chiu HY, Sun GH, Chen SY, et al: Pre-existing Fas ligand (FasL) in cancer cells elicits tumor-specific protective immunity, but delayed induction of FasL expression after inoculation facilitates tumor formation. Mol Carcinog 52: 705-714, 2013.
5. Ardolino M, Azimi CS, Iannello A, et al: Cytokine therapy reverses NK cell anergy in MHC-deficient tumors. J Clin Invest 124: 4781-4794, 2014.

6. Mkrtichyan M, Ghochikyan A, Davtyan H, et al: Cancer-testis antigen, BORIS based vaccine delivered by dendritic cells is extremely effective against a very aggressive and highly metastatic mouse mammary carcinoma. Cell Immunol 270: 188-197, 2011

7. Burdek M, Spranger S, Wilde S, et al: Three-day dendritic cells for vaccine development: antigen uptake, processing and presentation. J Transl Med 8: 90, 2010.

8. Parry AL, Clemson NA, Ellis J, et al: 'Multicopy multivalent' glycopolymer-stabilised gold nanoparticles as potential synthetic cancer vaccines. J Am Chem Soc 135: 9362-9365, 2013.

9. Hunn MK, Farrand KJ, Broadley KW, et al: Vaccination with irradiated tumor cells pulsed with an adjuvant that stimulates NKT cells is an effective treatment for glioma. Clin Cancer Res 18: 6446-6459, 2012

10. Teitz-Tennenbaum S, Li Q, Davis MA and Chang AE: Dendritic cells pulsed with keyhole limpet hemocyanin and cryopreserved maintain anti-tumor activity in a murine melanoma model. Clin Immunol 129: 482-491, 2008.

11. Chiang CL, Kandalaft LE, Tanyi J, et al: A dendritic cell vaccine pulsed with autologous hypochlorous acid-oxidized ovarian cancer lysate primes effective broad antitumor immunity: from bench to bedside. Clin Cancer Res 19: 4801-4815, 2013.

12. Obata C, Zhang M, Moroi Y, et al: Formalin-fixed tumor cells effectively induce antitumor immunity both in prophylactic and therapeutic conditions. J Dermatol Sci 34: 209-219, 2004.

13. Clay TM, Mosca PJ, Lyerly HK and Morse MA: Whole-tumor-cell vaccines. In: Handbook of Cancer Vaccines. Morse MA, Clay TM and Lyerly HK (eds). Springer, pp249-251, 2004.

14. Koido S, Homma S, Okamoto M, et al: Fusions between dendritic cells and whole tumor cells as anticancer vaccines. Oncoimmunology 2: e24437, 2013.

15. Yu P and Fu YX: Tumor-infiltrating T lymphocytes: friends or foes? Laboratory Invest 86: 231-245, 2006.

16. Sharma P, Shen Y, Wen S, et al: CD8 tumor-infiltrating lymphocytes are predictive of survival in muscle-invasive urothelial carcinoma. Proc Natl Acad Sci USA 104: 3967-3972, 2007.

17. Clemente CG, Mihm MC Jr, Bufalino R, et al: Prognostic value of tumor infiltrating lymphocytes in the vertical growth phase of primary cutaneous melanoma. Cancer 77: 1303-1310, 1996.

18. Aerts JG and Hegmans JP: Tumor-specific cytotoxic T cells are crucial for efficacy of immunomodulatory antibodies in patients with lung cancer. Cancer Res 73: 2381-2388, 2013.

19. Dudley ME, Wunderlich JR, Robbins PF, et al: Cancer regression and autoimmunity in patients after clonal repopulation with antitumor lymphocytes. Science 298: 850-854, 2002.

20. Sakaguchi S, Yamaguchi T, Nomura T and Ono M: Regulatory T cells and immune tolerance. Cell 133: 775-787, 2008.

21. Liu WK, Ho JC, Cheung FW, et al: Apoptotic activity of betulinic acid derivatives on murine melanoma B16 cell line. Eur J Pharmacol 498: 71-78, 2004.

22. Eggermont AM: Therapeutic vaccines in solid tumours: can they be harmful? Eur J Cancer 45: 2087-2090, 2009.

23. Pajtasz-Piasecka E and Indrová M: Dendritic cell-based vaccines for the therapy of experimental tumors. Immunotherapy 2: 257-268, 2010.

24. Hira SK, Mondal I and Manna PP: Combined immunotherapy with whole tumor lysate-pulsed interleukin-15-activated dendritic cells and cucurbitacin I promotes strong CD8(+) T-cell responses and cures highly aggressive lymphoma. Cytotherapy 17: 647-664, 2015.

25. Manzo T, Michelini RH, Sturmheit T, et al: Tumor-targeting vaccination instructs graft-vs.-tumor immune responses. Oncoimmunology 2: e25996, 2013.

26. Wu J, Fu W, Luo J and Zhang T: Expression and purification of human endostatin from Hansenula polymorpha A16. Protein Expr Purif 42: 12-19, 2005.

27. Kósa JP, Horváth P, Wölfling J, et al: CYP24A1 inhibition facilitates the anti-tumor effect of vitamin D3 on colorectal cancer cells. World J Gastroenterol 19: 2621-2628, 2013.

28. Zamarron BF and Chen WJ: Dual roles of immune cells and their factors in cancer development and progression. Int J Biol Sci 7: 651-658, 2011. 